\title{
StePAR: an automatic code to infer stellar atmospheric parameters
}

\author{
H. M. Tabernero ${ }^{1,2}$, E. Marfil ${ }^{3}$, D. Montes ${ }^{3}$, and J. I. González Hernández ${ }^{4,5}$ \\ ${ }^{1}$ Centro de Astrobiología (CSIC-INTA), Carretera de Ajalvir km 4, Torrejón de Ardoz, 28850 Madrid, Spain \\ 2 Instituto de Astrofísica e Ciências do Espaço, Universidade do Porto, CAUP, Rua das Estrelas, 4150-762 Porto, Portugal \\ e-mail: hugo.tabernero@astro.up.pt \\ 3 Departamento de Física de la Tierra y Astrofísica \& IPARCOS-UCM (Instituto de Física de Partículas y del Cosmos de la UCM), \\ Facultad de Ciencias Físicas, Universidad Complutense de Madrid, 28040 Madrid, Spain \\ ${ }^{4}$ Instituto de Astrofísica de Canarias (IAC), 38205 La Laguna, Tenerife, Spain \\ 5 Universidad de La Laguna (ULL), Departamento de Astrofísica, 38206 La Laguna, Tenerife, Spain
}

Received 14 March 2019 / Accepted 14 July 2019

\begin{abstract}
Context. STEPAR is an automatic code written in Python 3.X designed to compute the stellar atmospheric parameters $T_{\text {eff }}, \log g$, [Fe/H], and $\xi$ of FGK-type stars by means of the equivalent width (EW) method. This code has already been extensively tested in different spectroscopic studies of FGK-type stars with several spectrographs and against thousands of Gaia-ESO Survey UVES U580 spectra of late-type, low-mass stars as one of its 13 pipelines.

Aims. We describe the code that we tested against a library of well characterised Gaia benchmark stars. We also release the code to the community and provide the link for download.

Methods. We carried out the required EW determination of Fe I and Fe II spectral lines using the automatic tool TAME. STEPAR implements a grid of MARCS model atmospheres and the MOOG radiative transfer code to compute stellar atmospheric parameters by means of a Downhill Simplex minimisation algorithm.

Results. We show the results of the benchmark star test and also discuss the limitations of the EW method, and hence the code. In addition, we find a small internal scatter for the benchmark stars of $9 \pm 32 \mathrm{~K}$ in $T_{\text {eff }}, 0.00 \pm 0.07 \operatorname{dex}$ in $\log g$, and $0.00 \pm 0.03$ dex in $[\mathrm{Fe} / \mathrm{H}]$. Finally, we advise against using STEPAR on double-lined spectroscopic binaries or spectra with $R<30000, S / N<20$, or $v \sin i>15 \mathrm{~km} \mathrm{~s}^{-1}$, and on stars later than $\mathrm{K} 4$ or earlier than $\mathrm{F} 6$.
\end{abstract}

Key words. techniques: spectroscopic - methods: data analysis - stars: fundamental parameters

\section{Introduction}

The characterisation of stellar spectra is a matter of utmost importance to several fields in modern astrophysics. It provides for the study and better understanding of the different constituents of our galaxy in terms of both individual and large-scale properties of target objects.

For this reason, stellar spectroscopy is a powerful tool that is being widely used in observational surveys, such as the APO Galactic Evolution Experiment (APOGEE, Dawson et al. 2013), the GALactic Archeology with HERMES (GALAH, De Silva et al. 2015), the LAMOST Experiment for Galactic Understanding and Exploration (LEGUE, Deng et al. 2012), the RAdial Velocity Experiment (RAVE, Kunder et al. 2017), the Sloan Extension for Galactic Understanding and Exploration (SEGUE, Lee et al. 2008), the Gaia-ESO Survey (GES, Gilmore et al. 2012), and the WHT Enhanced Area Velocity Explorer (WEAVE, Dalton et al. 2018).

These large surveys have been specially designed to yield full sets of stellar parameters for as many stars as possible by means of automated methods that ensure the homogeneity of the results. These parameters include the effective temperature, $T_{\text {eff }}$; the surface gravity, $\log g$; the metallicity, $[\mathrm{M} / \mathrm{H}]$; and the microturbulent velocity, $\xi$.

In this regard, late-type, low-mass stars of FGK spectral types remain some of the most interesting targets on account of their ubiquity. Furthermore, the optical spectra of these stars have many iron features that are very sensitive to the stellar atmospheric parameters.

The computation of the stellar atmospheric parameters of FGK stars under spectroscopic scrutiny is often carried out by means of two different methods: spectral synthesis and equivalent width (EW). The former uses theoretical synthetic spectra in order to find the best match to a target observed spectrum, whereas the latter uses the strength of several spectral lines to find the set of stellar atmospheric parameters that best reproduces the measured EWs. Recent thorough reviews of these techniques can be found in Allende Prieto (2016), Nissen \& Gustafsson (2018), Jofré et al. (2019), and Blanco-Cuaresma (2019).

There are many implementations of these two methods that are publicly available to the community. Among the spectral synthesis implementations are the APOGEE pipeline (ASCAP, García Pérez et al. 2016) and Spectroscopy Made Easy (SME, Piskunov \& Valenti 2017; Valenti \& Piskunov 1996), whereas the EW method is implemented in tools such as FAMA (Magrini et al. 2013), GALA (Mucciarelli et al. 2013), BACCHUS (Masseron et al. 2016), and SPECIES (Soto \& Jenkins 2018). Remarkably, there are also general-purpose toolkits such as the integrated Spectroscopic framework (iSpec, see Blanco-Cuaresma et al. 2014a) and FASMA (Andreasen et al. 2017; Tsantaki et al. 2018) that can compute the stellar atmospheric parameters of any given star using both methods.

In general, spectral synthesis methods are based on a $\chi^{2}$ minimisation algorithm, in turn based on a pre-computed grid of 
atmospheric models (see Valenti \& Piskunov 1996; García Pérez et al. 2016; Tsantaki et al. 2018). The theoretical spectra, which may sometimes be split up into spectral regions of interest (see e.g. Tsantaki et al. 2014), are finally compared with the observations to find the atmospheric model that best fits the data. This approach can also be found in González Hernández et al. (2004) and Allende Prieto et al. (2006).

On the other hand, the EW method employs the standard technique based on the iron ionisation and excitation balance, taking advantage of the high sensitivity of the strength (i.e. the $\mathrm{EW}$ ) of $\mathrm{Fe}$ I and Fe II lines to the variation of the stellar atmospheric parameters. This approach rests on the curves of growth that link, by means of the Saha and Boltzmann equations, the observed EW to the column density of the chemical species that causes the line in the stellar spectrum. Further details of these two equations can be found in Hubeny \& Mihalas (2014), among others. This method has already been applied to several studies found in the literature (see e.g. Ghezzi et al. 2010; Santos et al. 2004; Sousa et al. 2008).

The required EW determination of the Fe lines can be carried out either automatically or manually. There are some automatic tools designed for this task, such as ARES (Sousa et al. 2007), DAOSPEC (Stetson \& Pancino 2008), and TAME (Kang \& Lee 2012). All these tools accept some input parameters that can be fine-tuned depending on the quality of the target spectrum under analysis (e.g. the position of the stellar pseudo-continuum, the list of spectral lines to be measured, the parameters that constrain the detection of spectral lines according to the spectral resolution). In this regard, we note that any given linelist is generally assembled from the analysis of a template star (usually the Sun; e.g. Santos et al. 2004; Sousa et al. 2008). However, in some cases the template star may be different, for example a cool K-type star (Tsantaki et al. 2013) or a giant star (Hekker \& Meléndez 2007). The selected lines must be as unblended as possible to avoid the contamination of neighbouring lines that could potentially affect the EW measurements.

In this work we present a full description of the automatic code STEPAR, written in Python 3.X, which is based on the EW method. This code has already been applied to the careful study of FGKtype stars (González Hernández et al. 2012; Tabernero et al. 2012, 2017; Tabernero 2014; Jofré et al. 2017; Montes et al. 2018) and has also been extensively used to automatically analyse hundreds of Gaia-ESO UVES spectra since it is one of the 13 pipelines that characterise the UVES U580 spectra of late-type, low-mass stars (see Smiljanic et al. 2014; Lanzafame et al. 2015).

A concise description of the complete method is found in Sect. 2. Detailed explanations of the STEPAR internal workflow are given in Sect. 3. In Sect. 4 we discuss our results and compare them to those obtained in previous works, and to evolutionary tracks. Finally, in Sect. 5 we present the limitations of the EW method, and hence STEPAR.

\section{The StePar code}

\subsection{StePAR ingredients}

The basic ingredients that STEPAR needs to derive the stellar atmospheric parameters $T_{\text {eff }}, \log g,[\mathrm{Fe} / \mathrm{H}]$, and $\xi$ are the following (see Sect. 2.2 for full explanation):

(i) A grid of stellar atmospheric models: MARCS mode atmospheres (Gustafsson et al. 2008);

(ii) A code to solve the radiative transfer problem under the assumption of local thermodynamic equilibrium (LTE): the MOOG code (Sneden 1973); (iii) A list of Fe I and Fe II spectral lines along with their atomic parameters;

(iv) A programme to measure the required EWs for later use: TAME (Kang \& Lee 2012);

(v) An optimisation algorithm: the Downhill Simplex method (Press et al. 2002).

\subsection{StePAR workflow}

The stellar atmospheric parameters of FGK-type stars, namely $T_{\text {eff }}, \log g, \xi$, and $[\mathrm{Fe} / \mathrm{H}]$, can be derived in an automated fashion with STEPAR ${ }^{1}$. Its workflow is shown in Fig. 1. In the standard STEPAR version presented here, we employed the 2017 version of the MOOG code (via the abfind driver, see Sneden 1973) and a grid of plane-parallel and spherical MARCS model atmospheres (Gustafsson et al. 2008), although other model grids can be used alongside STEPAR (see Tabernero et al. 2012, 2017; Montes et al. 2018). For lower gravities $(\log g<3.5)$ we used the spherical grid, whereas we employed the non-spherical grid for greater gravities $(\log g \geq 3.5)$. Although MOOG treats the MARCS spherical atmospheric models as if they were planeparallel, Heiter \& Eriksson (2006) proved that this potential inconsistency is negligible. However, since the MARCS grid is finite, StePAR includes an interpolation subroutine, based on the Python Scipy library, which draws on the prior knowledge of the desired model and its neighbouring grid models to interpolate between them (Barber et al. 1996).

STEPAR needs a MOOG-compliant EW file as input, which can be provided by the user in the proper format using an automatic measurement tool. This MOOG-input file must contain the following atomic data for each line considered in the analysis:

(i) Central wavelength of the line, in $\AA$;

(ii) A number that indicates the atomic number and ionisation stage of the chemical species that causes the line (26.0 and 26.1 in the case of $\mathrm{Fe}_{\mathrm{I}}$ and $\mathrm{Fe}$ II lines, respectively);

(iii) The excitation potential, $\chi$, in $\mathrm{eV}$;

(iv) The oscillator strength, $\log g f$;

(v) The EW of the line in $\mathrm{m} \AA$.

To perform our analysis we opted for the automatic code $\mathrm{TAME}^{2}$ (Kang \& Lee 2012), which can be run in either an automated or manual mode. Its manual mode has an interface that allows some user control over the EW measurements to check problematic spectra when needed. We followed the approach of Kang \& Lee (2012) to adjust the rejt parameter of TAME according to the $\mathrm{S} / \mathrm{N}$ of each spectrum. The other TAME parameters we employed were

(i) smoother $=4$, the recommended parameter for smoothing the derivatives used for line identification;

(ii) space $=3$, the wavelength interval (in $\AA$ ) from each side of the central line to perform the EW computation;

(iii) lineresol $=0.1$, the minimum distance (in $\AA$ ) between two lines for TAME to resolve them;

(iv) miniline $=2$, the minimum $\mathrm{EW}$ that will be printed in the output.

Further details on the TAME parameters can be found in Kang \& Lee (2012). In addition, we only considered measured lines with $10 \mathrm{~m} \AA<\mathrm{EW}<120 \mathrm{~m} \AA$ to avoid problems with line profiles of very intense lines and tentatively bad EW measurements of extremely weak lines. For benchmark stars that have two high

1 StePAR is available at https://github.com/hmtabernero/ StePar under the two-clause BSD license.

2 TAME can be downloaded from http://astro.snu.ac.kr/ $\sim$ wskang/tame/ 


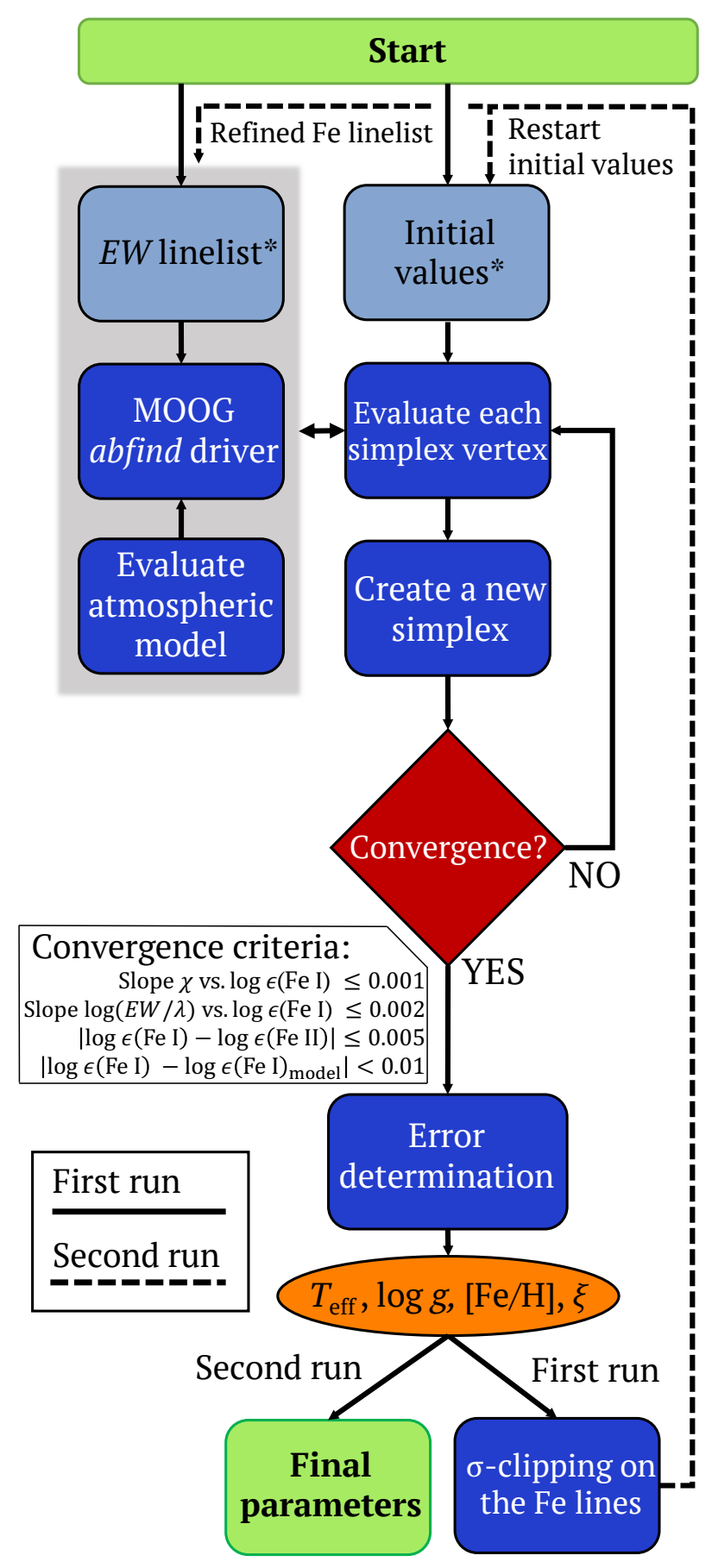

Fig. 1. StePAR workflow diagram. For any given star under analysis, the code performs two simplex runs. During the second simplex run, the initial values, which are initially set to the solar canonical values, are reset to the values obtained during the first run. Likewise, the EW linelist is refined according to the $\sigma$ clipping procedure on the Fe I lines. After the second run, the code halts execution and yields the final solution for the star. See Sect. 2.2 for explanation.

signal-to-noise ratio $(\mathrm{S} / \mathrm{N})$ spectra available, we applied an additional filter on the Fe I, II lines and rejected the ones that might have a differential equivalent width beyond three times the standard deviation of the EW differences between the corresponding Fe I, II lines measured on each of those two spectra.

As damping prescription, we used the Anstee-BarcklemO’Mara (ABO, see Barklem et al. 1998) data (if available)
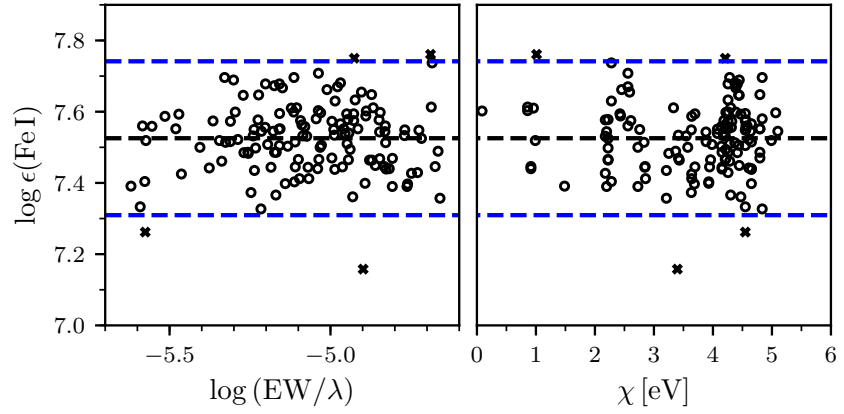

Fig. 2. StePAR inner $3 \sigma$ clipping of the Fe I lines on the NARVAL spectrum of the Sun. $\log \epsilon\left(\mathrm{Fe}_{\mathrm{I}}\right)$ stands for the $\mathrm{Fe}$ abundance returned by the Fe lines, while $\log (\mathrm{EW} / \lambda)$ represents their reduced EWs. Black crosses depict the rejected $\mathrm{Fe}_{\mathrm{I}}$ lines. The dashed black lines represent a linear fit to the points, whereas the dashed blue lines are located at the $3 \sigma$ level.

through option 1 of MOOG. The atmospheric parameters can then be inferred from previously assembled $\mathrm{Fe}$ I-Fe II linelists.

The minimisation procedure of STEPAR is the Downhill Simplex algorithm (Press et al. 2002), which tries to minimise a quadratic form composed of the excitation and ionisation equilibrium conditions to find the best parameters of the target star. This minimisation algorithm can reach convergence in very few iterations, and it is so fast that it is the optimisation method of choice, for instance, for the ASCAP pipeline in APOGEE (Dawson et al. 2013). Since it does not use derivatives, they have to be estimated numerically, as in the Levenberg-Marquardt method. If we let $\log \epsilon\left(\mathrm{Fe}_{\mathrm{I}}\right)$, and $\log \epsilon(\mathrm{Fe}$ II $)$ stand for the $\mathrm{Fe}$ abundance returned by the $\mathrm{Fe}_{\mathrm{I}}$ and $\mathrm{Fe}_{\mathrm{II}}$ lines, respectively, and $\log (E W / \lambda)$ be their reduced equivalent width, STEPAR iterates until the slopes of $\chi$ versus $\log \epsilon\left(\mathrm{Fe}_{\mathrm{I}}\right)$ and $\log (E W / \lambda)$ versus $\log \epsilon(\mathrm{Fe} \mathrm{I})$ are virtually zero (i.e. excitation equilibrium, and imposing ionisation equilibrium) so that $\log \epsilon\left(\mathrm{Fe}_{\mathrm{I}}\right)=$ $\log \epsilon(\mathrm{Fe}$ II $)$. Throughout this procedure, we checked that the $[\mathrm{Fe} / \mathrm{H}]$ obtained from the iron lines is always compatible with the metallicity of the input atmospheric model. The actual convergence criteria of STEPAR, as shown in Fig. 1, are the following:

(i) Slope $\xi$ vs. $\log \epsilon(\mathrm{Fe}$ I $) \leq 0.001$;

(ii) Slope $\log \mathrm{EW} / \lambda$ vs. $\log \epsilon\left(\mathrm{Fe}_{\mathrm{I}}\right) \leq 0.002$;

(iii) $\mid \log \epsilon\left(\mathrm{Fe}_{\mathrm{I}}\right)-\log \epsilon(\mathrm{Fe}$ II $) \mid \leq 0.005$;

(iv) $\left|\log \epsilon\left(\mathrm{Fe}_{\mathrm{I}}\right)-\log \epsilon\left(\mathrm{Fe}_{\mathrm{I}}\right)_{\text {model }}\right|<0.01$.

For each target spectrum, STEPAR performs two simplex runs, which in turn individually entail a full parameter determination using the Downhill Simplex optimisation method. The first run deals with the EWs file as initially measured by TAME. Next, the best model that is found in this first run by the optimisation routine is evaluated. STEPAR then performs a $3 \sigma$ clipping procedure on the $\mathrm{Fe}_{\mathrm{I}}$ abundance values obtained from the Fe I lines so that we can remove the outliers, if any, due to "wrong" EW measurements that could potentially invalidate the analysis. The second run is finally launched on a new input EW file that does not contain the rejected lines. An example of this $3 \sigma$ clipping procedure is shown in Fig. 2. As to execution time, STEPAR takes between 2 and 5 min per star to perform this whole procedure, depending on its actual position in the FGK parameter space.

To feed this minimisation process, the canonical solar values are used as initial input values $\left(T_{\text {eff }}=5777 \mathrm{~K}, \log g=4.44 \mathrm{dex}\right.$, $\left.\xi=1 \mathrm{~km} \mathrm{~s}^{-1}\right)$. However, STEPAR is able to reach a solution even if the problem star is different from the Sun (e.g. a metal-poor giant). In other words, the final solution for any given star is independent of the initial set of parameters employed. 
Finally, the uncertainties in the stellar parameters are determined as follows:

(i) For the micro-turbulence, we slightly change the value of $\xi$ until the slope of $\log \epsilon(\mathrm{Fe} \mathrm{I})$ vs. $\log (\mathrm{EW} / \lambda)$ varies within its own error, divided by the square root of the number of $\mathrm{Fe}$ I lines.

(ii) The effective temperature is varied until the slope of $\log \epsilon\left(\mathrm{Fe}_{\mathrm{I}}\right)$ vs. $\chi$ increases to the error on the slope, divided by the square root of the number of Fe I lines. By increasing $\xi$ on its error, we recompute the effective temperature. These two sources of error are added in quadrature.

(iii) The surface gravity is then varied until the Fe II abundance increases by a quantity equal to the standard deviation divided by the square root of the number of Fe II lines. All the previous errors in $\xi$ and $T_{\text {eff }}$ are taken into account by varying these quantities separately, thus recomputing the gravity. These differences are later added in quadrature.

(iv) Finally, to determine the error in the Fe abundance the stellar atmospheric parameters are varied in their respective uncertainties, which enables the combination of all the Fe I, II variations due to the stellar parameters uncertainties and the standard deviation of the Fe I, II abundances in quadrature.

\section{Testing the code}

\subsection{Selection of the Fe I, ॥ linelists}

The EW method requires a significantly large selection of reliable $\mathrm{Fe}$ I and $\mathrm{Fe}$ II lines. In principle, reliable lines are meant to be clean spectral lines that are not strongly affected by line blending of neighbouring lines or conspicuous spectral features. All the same, the available atomic data of these clean lines may not be precise enough for a trustworthy analysis. The main problem stems from the tabulated values of the transition probability per unit time of the spectral lines, $\log g f$. Some authors avoid this problem by calibrating this value for each line (see e.g. Santos et al. 2004; Sousa et al. 2008; Neves et al. 2009), normally by means of an inverse solar analysis where they vary the $\log g f$ value for a given line until they recover the corresponding solar abundance value. These are called the astrophysical $\log g f \mathrm{~s}$.

The Gaia-ESO linelist (Heiter et al. 2015a) was originally extracted from a variety of sources with the aim of finding the best atomic parameters available. It contains around $560 \mathrm{Fe}$ I, II features whose parameters were mostly taken from the Vienna Atomic Line Database (VALD ${ }^{3}$, Ryabchikova et al. 2015). The prime goal was to compile a trustworthy selection of lines to compute high-precision stellar parameters.

However, given the diversity of stars across the Milky Way (metal-poor dwarfs and giants, solar-type stars, metal rich giants, etc.) in the Gaia-ESO Survey, it soon became apparent that one linelist may fall short for the analysis of any given star. Hence, the analysis of any stellar sample under STEPAR is set to rely on four template stars from which four different lists of Fe I, II lines are assembled: the Sun, HD 22879, $\xi$ Hya, and Arcturus. The corresponding linelists of Fe I and Fe II can be found in Tables A.3 and A.4, respectively. These four template stars, which fully cover the FGK parameter space (as explained below) help us classify any star prior to the analysis with STEPAR.

This division of the parameter space meets the following criteria. In terms of metallicity, we distinguish between metal-rich stars, $[\mathrm{Fe} / \mathrm{H}] \geq-0.30$, and metal-poor stars, $-0.30>[\mathrm{Fe} / \mathrm{H}] \geq$ -1.50 . In terms of surface gravity, we make a distinction between the giant regime, $\log g<4.00$, and the dwarf regime,

\footnotetext{
http://www . astro.uu.se/ vald/
}

Table 1. Linelist template stars and their reference stellar atmospheric parameters from Heiter et al. (2015b), with updated values from Jofré et al. (2019).

\begin{tabular}{llccc}
\hline \hline Star & List & $T_{\text {eff }}[\mathrm{K}]$ & $\log g[\mathrm{dex}]$ & {$[\mathrm{Fe} / \mathrm{H}][\mathrm{dex}]$} \\
\hline Sun & MRD & $5777 \pm 1$ & $4.44 \pm 0.01$ & $0.03 \pm 0.01$ \\
HD 22879 & MPD & $5868 \pm 89$ & $4.27 \pm 0.03$ & $-0.86 \pm 0.05$ \\
$\xi$ Hya & MRG & $5044 \pm 40$ & $2.87 \pm 0.02$ & $0.16 \pm 0.20$ \\
Arcturus & MPG & $4286 \pm 35$ & $1.60 \pm 0.20$ & $-0.52 \pm 0.08$ \\
\hline
\end{tabular}

$\log g \geq 4.00$. These partitions mean that the global parameter space is divided into four different regions: metal-rich dwarfs (MRD), metal-poor dwarfs (MPD), metal-rich giants (MRG), and metal-poor giants (MPG). Because of their scarcity, we decided to set aside the extremely metal-poor stars with $[\mathrm{Fe} / \mathrm{H}]<-1.50$. The general scheme of this division $(\mathrm{MRD}$, MPD, MRG, MPG) is shown in Table 1.

As already mentioned, the analysis of any given star is done blindly, that is, its stellar parameters are not known beforehand. Hence, it is not known a priori which linelist corresponds to any given star. In order to overcome this issue, we measured the lines from all four linelists and did a first-pass with STEPAR so we could finally assign a linelist to each star depending on the parameters obtained. This preliminary step allowed us to run STEPAR with the corresponding linelist to get the final solution for the star.

\subsection{Gaia benchmark test}

Every spectroscopic study requires a reference point to assess the validity of the obtained results. Even though the Sun is widely used in the literature as a common reference, the use of one single star as a central point of reference provides no evidence for how a given method works in different regions of the parameter space.

In this regard, the Gaia benchmark stars were originally meant as calibrators to test the different approaches to the analysis. The availability of specific information for these stars was the key to determining their stellar atmospheric parameters independently from spectroscopy (Heiter et al. 2015b). In this sense, the Gaia benchmark stars represent a cornerstone when it comes to weighing the impact of the general limitations (e.g. wavelength coverage, linelists employed, resolution) inherent to any spectroscopic method that aims at the computation of such parameters.

Ideally, with a common background, any set of tools using the same data should converge to the same atmospheric parameters. However, given that every method takes into account a different set of spectral lines, this inevitably leads to slightly different stellar parameters. Although, in general, these differences are mostly dependent on the radiative transfer code, the stellar atmospheric models, the specific method, and the input data, among the GES nodes this dependence mostly comes down to the input data (measurement of EWs, S/N of the spectra, the local continuum normalisation, etc.) and the method that each node takes into consideration.

These stars, 23 in total, were taken from the stellar library ${ }^{4}$ described in Blanco-Cuaresma et al. (2014b), which covers the optical region $4800-6800 \AA$. The stars span a wide range in the

\footnotetext{
4 The spectra of these stars can be downloaded from https://wwW .
} blancocuaresma.com/s/benchmarkstars 

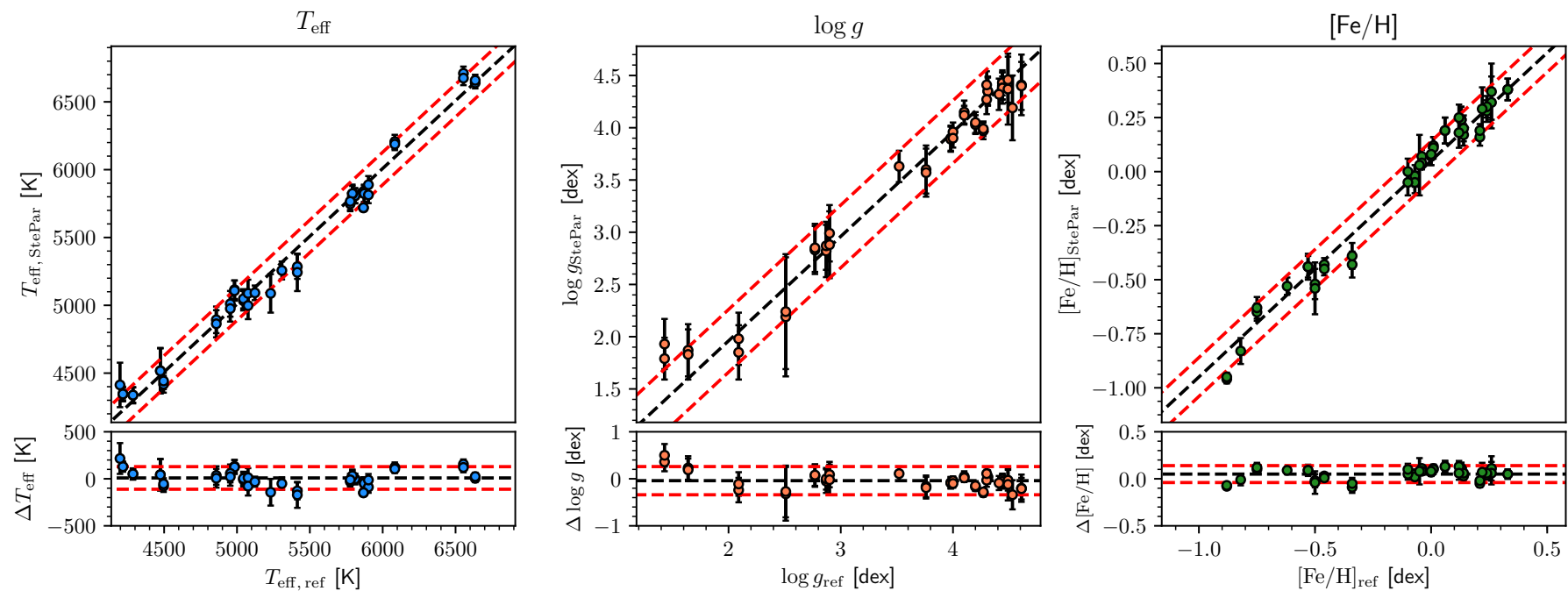

Fig. 3. StePar results for the Gaia benchmark stars plotted against the literature values taken from Heiter et al. (2015b), with updated values from Jofré et al. (2019). Upper panels: one-to-one correspondence, whereas bottom panels: absolute differences. Using the values in Table 3, the dashed black lines in the upper panels correspond to a one-to-one relationship, shifted following the average differences in each parameter, whereas in the bottom panels they are centred on the average differences. The dashed red lines in all panels correspond to a margin of $120 \mathrm{~K}$, $0.30 \mathrm{dex}$, and $0.09 \mathrm{dex}$ in $T_{\mathrm{eff}}, \log g$, and $[\mathrm{Fe} / \mathrm{H}]$, respectively, according to the $\sigma$ values found in the differences in each parameter.

Table 2. Number of $\mathrm{Fe}_{\mathrm{I}}$ and $\mathrm{Fe}$ II in each of the four linelists used in this work.

\begin{tabular}{lcccc}
\hline \hline Element & MRD & MPD & MRG & MPG \\
\hline Fe I $_{\text {I }}$ & 146 & 127 & 113 & 115 \\
Fe $_{\text {II }}$ & 12 & 13 & 11 & 6 \\
\hline \#stars & 8 & 4 & 7 & 4 \\
\hline
\end{tabular}

Notes. The wavelength coverage of all four lists is $4800-6800 \AA$, in line with that of the spectra under analysis. We also display the number of stars in each category.

parameter space (Heiter et al. 2015b; Jofré et al. 2015), which allowed us to test the reliability of the results given by STEPAR (see Table A.1). The internal consistency of the code was tested by deriving stellar atmospheric parameters from one or two high $\mathrm{S} / \mathrm{N}$ spectra of the same star (see Table A.2).

\section{Discussion}

Although different spectra of the same object should theoretically result in exactly the same stellar atmospheric parameters, we note slight deviations in our analysis. The mean differences for the benchmark stars are $9 \pm 32 \mathrm{~K}$ in $T_{\text {eff }}, 0.00 \pm 0.07 \mathrm{dex}$ in $\log g$, and $0.00 \pm 0.03 \mathrm{dex}$ in $[\mathrm{Fe} / \mathrm{H}]$.

These internal differences are mostly due to the quality of the individual spectra. The average uncertainties for the benchmark stars are $75 \mathrm{~K}$ in $T_{\text {eff }}, 0.21 \mathrm{dex}$ in $\log g$, and $0.06 \mathrm{dex}$ in $[\mathrm{Fe} / \mathrm{H}]$. Furthermore, the average uncertainties are greater than the scatter that arises from the analysis of different spectra of the same object, as expected. Other sources of uncertainty might arise from systematic effects inherent to any methodology.

We compared the values of the stellar parameters for each spectrum to the reference values, as shown in Fig. 3. We found the following differences, all of which might be systematic: $\Delta T_{\text {eff }}=9 \pm 89 \mathrm{~K}, \Delta \log g=-0.04 \pm 0.18 \mathrm{dex}$, and $\Delta[\mathrm{Fe} / \mathrm{H}]=$ $0.05 \pm 0.06$ dex. At first glance, there are no notable differences at the $1-2 \sigma$ level. Additionally, in Fig. 4 we plot the line iron abundance retrieved by $\mathrm{STEPAR}_{\mathrm{T}}$ for the final solution of the four reference stars.

However, since systematic trends may still remain hidden, we performed 10000 Monte Carlo simulations on our data (as in Tabernero et al. 2018) in the hope of assessing possible sources of tentatively systematic offsets. We took each atmospheric parameter $\left(T_{\text {eff }}, \log g\right.$, and $\left.[\mathrm{Fe} / \mathrm{H}]\right)$ to check any possible correlations by means of the Pearson and Spearman correlation coefficients, which are a measure of the correlation between any two given variables. Specifically, the Pearson coefficient is a classical correlation indicator, whereas the Spearman coefficient is a more robust non-parametric estimator of the statistical dependence of any two given variables (for more details, see e.g. Press et al. 2002). In Table 3 we clearly show that no systematic trends are present above the $2 \sigma$ level (i.e. within $95 \%$ confidence interval). These results are shown in Figs. 3 and A.1. Interestingly enough, even if the offsets in effective temperature and surface gravity are noticeable, the offset in the iron abundance is negligible (see Fig. 3). This is probably due to the fact that STEPAR produces self-consistent stellar parameters. For example, a deviation on the effective temperature can be compensated by the other parameters.

Finally, we assessed the performance of STEPAR in different regions of the parameter space. In Fig. 5 we plot a Kiel diagram (i.e. $\log g$ vs. $\log T_{\text {eff }}$ ) including the latest Yale-Potsdam Stellar Isochrones (YaPSI; Spada et al. 2017). In light of this figure, we do not find any major inconsistencies with the parameter space encompassed by the isochrones in general terms. However, we note that our method does not reproduce the gravity of $\mathrm{K}$ stars, as they should have higher values. The former result is not entirely unexpected (Tabernero et al. 2012), and might be due to an ionisation imbalance problem (Tsantaki et al. 2019). We still find higher effective temperatures for the F-type dwarfs, although they deviate less than in previous works (i.e. Tabernero et al. 2017; Montes et al. 2018). Finally, in Table 4 we show how STEPAR performs equally well in the four regions of the FGK parameter space defined in this work, i.e. MRD, MPD, MRG, and MPG, although the MRD and MPD linelists produce slightly larger errors than the rest. Interestingly enough, the errors on 

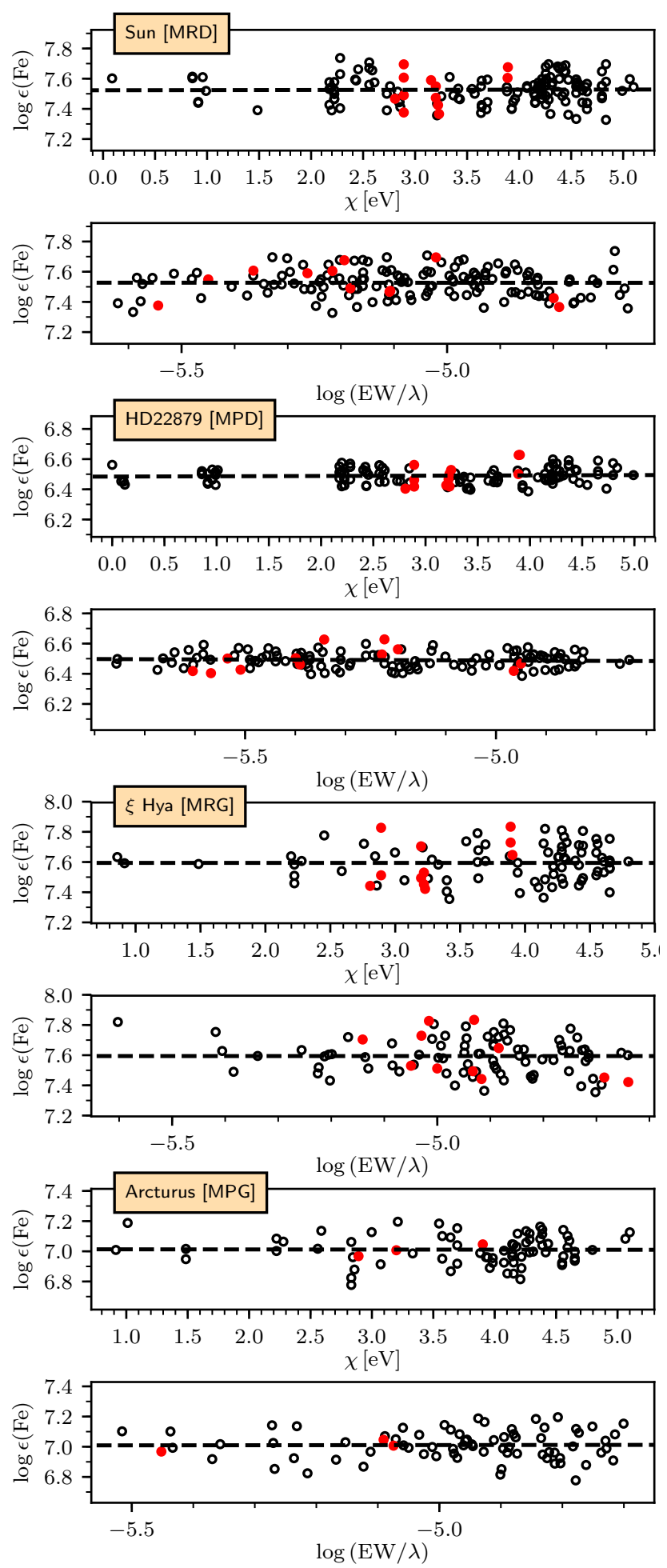

Fig. 4. From top to bottom: line iron abundance retrieved by STEPAR for the final solution of the four reference stars: the Sun (NARVAL), HD 22879 (NARVAL), $\xi$ Hya (ESPaDOns), and Arcturus (UVES). $\log \epsilon\left(\mathrm{Fe}_{\mathrm{I}}\right)$ stands for the $\mathrm{Fe}$ abundance returned by the Fe lines, while $\log (\mathrm{EW} / \lambda)$ is their reduced EWs. Unfilled black dots represent Fe I lines, whereas red dots are $\mathrm{Fe}$ II lines. The dashed black lines represent the least-squares fit to the data points.

surface gravity are larger in the giant regime than in the dwarf regime. For the metallicity we find a similar scatter in the four regions. Despite these differences, we find a good agreement with the reference values.

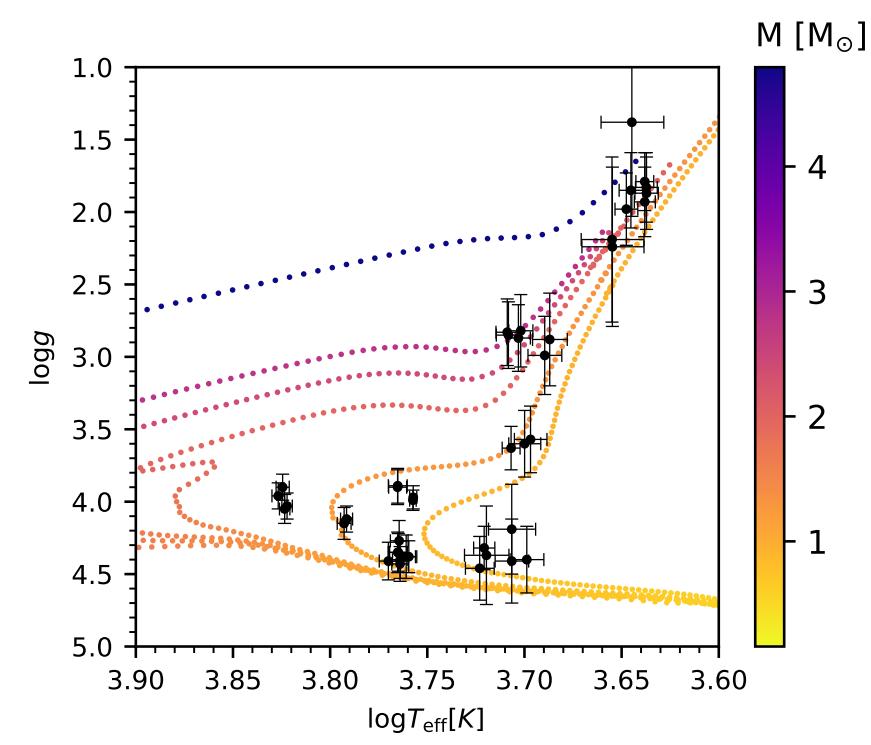

Fig. 5. Kiel diagram $\left(\log g\right.$ vs. $\left.\log T_{\text {eff }}\right)$ for all the spectra alongside the YaPSI isochrones for $0.1,0.4,0.6,1,4$, and $13 \mathrm{Ga}$ (for $Z=0.016$, see Spada et al. 2017).

Table 3. Summary of the Monte Carlo simulations performed using the stellar atmospheric parameters calculated in Sect. 3.

\begin{tabular}{lccc}
\hline \hline Parameter & Difference & $r_{\mathrm{p}}$ & $r_{\mathrm{S}}$ \\
\hline$T_{\text {eff }}[\mathrm{K}]$ & $9 \pm 120$ & $-0.12 \pm 0.10$ & $-0.15 \pm 0.10$ \\
$\log g$ [dex] & $-0.04 \pm 0.30$ & $-0.33 \pm 0.16$ & $-0.26 \pm 0.14$ \\
{$[\mathrm{Fe} / \mathrm{H}][\mathrm{dex}]$} & $0.05 \pm 0.09$ & $0.25 \pm 0.10$ & $0.16 \pm 0.11$ \\
\hline
\end{tabular}

Notes. Here we present the average difference on each parameter, along with the values of the Pearson $\left(r_{\mathrm{p}}\right)$ and the Spearman $\left(r_{\mathrm{S}}\right)$ correlation coefficients.

Table 4. Same information as shown in Table 3, but organised on a perlinelist basis, i.e. MRD, MPD, MRG, and MPG lists.

\begin{tabular}{lccc}
\hline \hline \multicolumn{4}{c}{ Difference } \\
\hline List & $T_{\text {eff }}[\mathrm{K}]$ & $\log g[\mathrm{dex}]$ & {$[\mathrm{Fe} / \mathrm{H}][\mathrm{dex}]$} \\
\hline MRD & $14 \pm 105$ & $0.05 \pm 0.19$ & $0.06 \pm 0.07$ \\
MPD & $-88 \pm 96$ & $0.16 \pm 0.18$ & $0.03 \pm 0.06$ \\
MRG & $44 \pm 122$ & $0.05 \pm 0.36$ & $0.08 \pm 0.08$ \\
MPG & $28 \pm 92$ & $0.15 \pm 0.31$ & $0.05 \pm 0.09$ \\
\hline
\end{tabular}

\section{Conclusions}

In this work, we have presented a robust code, STEPAR, which will be useful to the community when deriving the stellar atmospheric parameters of FGK-type stars under the EW method. This code has already been tested during the last few years against the vast quantities of Gaia-ESO high-resolution spectra. We have also tested STEPAR against a library of Gaia Benchmark stars. Although we find some differences with the reference parameters, they are not significant.

Finally, we want to address some general limitations of the EW method that should be taken into consideration when using STEPAR. First, the data must be of high enough quality to be analysed. Since low $\mathrm{S} / \mathrm{N}$ may translate into poor $\mathrm{EW}$ measurements, this means that we highly recommend placing a cut at $S / N<20$. In addition, we note the importance of placing a lower limit in 
spectral resolution at $\mathrm{R}=30000$ to prevent undesired line blending and suboptimal placement of the continuum level (Freeman \& Bland-Hawthorn 2002). Second, STEPAR cannot derive stellar atmospheric parameters of fast-rotating stars. Although the EWs are not in fact altered by rotation, the line profiles are affected by rotational broadening and may no longer fit a Gaussian profile properly. In this sense, blending of neighbouring lines to the one of interest can also make it nearly impossible to get a reliable EW estimate for a given line. In these cases, we advise against using STEPAR on any star with a rotational velocity higher than $15 \mathrm{~km} \mathrm{~s}^{-1}$. In addition, double-lined spectroscopic binaries should also be removed from any sample to avoid obtaining unreliable parameters. Finally, we do not recommend deriving stellar parameters with STEPAR for stars earlier than F6 and later than K4.

Acknowledgements. We would like to thank the anonymous referee for the insightful comments and suggestions that improved the manuscript of the paper. The authors acknowledge financial support from the Spanish Ministerio de Ciencia, Innovación y Universidades through projects AYA2016-79425-C3-1 (UCM), AYA2016-79425-C3-2 (CAB), and AYA2017-86389-P (IAC). E.M. acknowledges financial support from the Spanish Ministerio de Educación y Formación Profesional through fellowship FPU15/01476. J.I.G.H. acknowledges financial support from the Spanish MINECO (Ministry of Economy of Spain) under the 2013 Ramón y Cajal programme MINECO RyC-2013-14875. H.M.T also acknowledges financial support from the FCT - Fundação para a Ciência e a Tecnologia through national funds (PTDC/FIS-AST/28953/2017) and by FEDER - Fundo Europeu de Desenvolvimento Regional through COMPETE2020 - Programa Operacional Competitividade e Internacionalização (POCI-01-0145-FEDER-028953)

\section{References}

Allende Prieto, C. 2016, Liv. Rev. Sol. Phys., 13, 1

Allende Prieto, C., Beers, T. C., Wilhelm, R., et al. 2006, ApJ, 636, 804

Andreasen, D. T., Sousa, S. G., Tsantaki, M., et al. 2017, A\&A, 600, A69

Barber, C. B., Dobkin, D. P., \& Huhdanpaa, H. T. 1996, ACM Trans. Math. Soft. 22, 469

Barklem, P. S., Anstee, S. D., \& O’Mara, B. J. 1998, PASA, 15, 336

Blanco-Cuaresma, S. 2019, MNRAS, 486, 2075

Blanco-Cuaresma, S., Soubiran, C., Heiter, U., \& Jofré, P. 2014a, A\&A, 569, A111

Blanco-Cuaresma, S., Soubiran, C., Jofré, P., \& Heiter, U. 2014b, A\&A, 566, A98

Dalton, G., Trager, S., Abrams, D. C., et al. 2018, in Ground-based and Airborne Instrumentation for Astronomy VII, SPIE Conf. Ser., 10702, 107021B

Dawson, K. S., Schlegel, D. J., Ahn, C. P., et al. 2013, AJ, 145, 10

Deng, L.-C., Newberg, H. J., Liu, C., et al. 2012, Res. Astron. Astrophys., 12, 735

De Silva, G. M., Freeman, K. C., Bland-Hawthorn, J., et al. 2015, MNRAS, 449 2604

Freeman, K., \& Bland-Hawthorn, J. 2002, ARA\&A, 40, 487

García Pérez, A. E., Allende Prieto, C., Holtzman, J. A., et al. 2016, AJ, 151, 144
Ghezzi, L., Cunha, K., Smith, V. V., et al. 2010, ApJ, 720, 1290

Gilmore, G., Randich, S., Asplund, M., et al. 2012, The Messenger, 147, 25

González Hernández, J. I., Rebolo, R., Israelian, G., et al. 2004, ApJ, 609, 988

González Hernández, J. I., Ruiz-Lapuente, P., Tabernero, H. M., et al. 2012, Nature, 489, 533

Gustafsson, B., Edvardsson, B., Eriksson, K., et al. 2008, A\&A, 486, 951

Heiter, U., \& Eriksson, K. 2006, A\&A, 452, 1039

Heiter, U., Lind, K., Asplund, M., et al. 2015a, Phys. Scr., 90, 054010

Heiter, U., Jofré, P., Gustafsson, B., et al. 2015b, A\&A, 582, A49

Hekker, S., \& Meléndez, J. 2007, A\&A, 475, 1003

Hubeny, I., \& Mihalas, D. 2014, Theory of Stellar Atmospheres

Jofré, P., Heiter, U., Soubiran, C., et al. 2015, A\&A, 582, A81

Jofré, P., Heiter, U., Worley, C. C., et al. 2017, A\&A, 601, A38

Jofré, P., Heiter, U., \& Soubiran, C. 2019, ARA\&A, in press, [arXiv:1811.08041]

Kang, W., \& Lee, S.-G. 2012, MNRAS, 425, 3162

Kunder, A., Kordopatis, G., Steinmetz, M., et al. 2017, AJ, 153, 75

Lanzafame, A. C., Frasca, A., Damiani, F., et al. 2015, A\&A, 576, A80

Lee, Y. S., Beers, T. C., Sivarani, T., et al. 2008, AJ, 136, 2022

Magrini, L., Randich, S., Friel, E., et al. 2013, A\&A, 558, A38

Masseron, T., Merle, T., \& Hawkins, K. 2016, Astrophysics Source Code Library [record ascl:1605.004]

Montes, D., González-Peinado, R., Tabernero, H. M., et al. 2018, MNRAS, 479, 1332

Mucciarelli, A., Pancino, E., Lovisi, L., Ferraro, F. R., \& Lapenna, E. 2013, ApJ, 766, 78

Neves, V., Santos, N. C., Sousa, S. G., Correia, A. C. M., \& Israelian, G. 2009, A\&A, 497, 563

Nissen, P. E., \& Gustafsson, B. 2018, A\&ARv, 26, 6

Piskunov, N., \& Valenti, J. A. 2017, A\&A, 597, A16

Press, W. H., Teukolsky, S. A., Vetterling, W. T., \& Flannery, B. P. 2002, Numerical recipes in $\mathrm{C}++$ : the art of scientific computing

Ryabchikova, T., Piskunov, N., Kurucz, R. L., et al. 2015, Phys. Scr., 90, 054005

Santos, N. C., Israelian, G., \& Mayor, M. 2004, A\&A, 415, 1153

Smiljanic, R., Korn, A. J., Bergemann, M., et al. 2014, A\&A, 570, A122

Sneden, C. 1973, ApJ, 184, 839

Soto, M. G., \& Jenkins, J. S. 2018, A\&A, 615, A76

Sousa, S. G., Santos, N. C., Israelian, G., Mayor, M., \& Monteiro, M. J. P. F. G. 2007, A\&A, 469, 783

Sousa, S. G., Santos, N. C., Mayor, M., et al. 2008, A\&A, 487, 373

Spada, F., Demarque, P., Kim, Y. C., Boyajian, T. S., \& Brewer, J. M. 2017, ApJ, 838,161

Stetson, P. B., \& Pancino, E. 2008, PASP, 120, 1332

Tabernero, H. M. 2014, PhD Thesis, Departamento de Astrofísica, Facultad de Ciencias Físicas, Universidad Complutense de Madrid, Spain

Tabernero, H. M., Montes, D., \& González Hernández, J. I. 2012, A\&A, 547, A13

Tabernero, H. M., Montes, D., González Hernández, J. I., \& Ammler-von Eiff, M. 2017, A\&A, 597, A33

Tabernero, H. M., Dorda, R., Negueruela, I., \& González-Fernández, C. 2018, MNRAS, 476, 3106

Tsantaki, M., Sousa, S. G., Adibekyan, V. Z., et al. 2013, A\&A, 555, A150

Tsantaki, M., Sousa, S. G., Santos, N. C., et al. 2014, A\&A, 570, A80

Tsantaki, M., Andreasen, D. T., Teixeira, G. D. C., et al. 2018, MNRAS, 473, 5066

Tsantaki, M., Santos, N. C., Sousa, S. G., et al. 2019, MNRAS, 569

Valenti, J. A., \& Piskunov, N. 1996, A\&AS, 118, 595 
Appendix A: Additional material


Fig. A.1. StePar differences with respect to the reference values (Heiter et al. 2015b), where each symbol denotes a different spectrograph: NARVAL (circles); HARPS.GBOG (squares); HARPS.Archive (diamonds); UVES.POP (upward triangles); UVES (downward triangles); ESPaDOnS (crosses).

Table A.1. Reference stellar atmospheric parameters of the Gaia benchmark stars taken from Heiter et al. (2015b), with updated values from Jofré et al. (2019).

\begin{tabular}{ccccc}
\hline \hline Star & Spectral type & $\begin{array}{c}T_{\text {eff }} \\
{[\mathrm{K}]}\end{array}$ & $\begin{array}{c}\log g \\
{[\mathrm{dex}]}\end{array}$ & $\begin{array}{c}{[\mathrm{Fe} / \mathrm{H}]} \\
{[\mathrm{dex}]}\end{array}$ \\
\hline \multicolumn{5}{c}{ Metal-rich dwarfs (MRD) } \\
Procyon & F5IV-V & $6554 \pm 84$ & $4.00 \pm 0.02$ & $0.01 \pm 0.08$ \\
$\beta$ Vir & F9V & $6083 \pm 41$ & $4.10 \pm 0.02$ & $0.24 \pm 0.07$ \\
$\mu$ Ara & G3IV-V & $5902 \pm 66$ & $4.30 \pm 0.03$ & $0.35 \pm 0.13$ \\
18 Sco & G2Va & $5810 \pm 80$ & $4.44 \pm 0.03$ & $0.03 \pm 0.03$ \\
$\alpha$ Cen A & G2V & $5792 \pm 16$ & $4.31 \pm 0.01$ & $0.26 \pm 0.08$ \\
Sun & G2V & $5771 \pm 1$ & $4.44 \pm 0.00$ & $0.03 \pm 0.05$ \\
$\alpha$ Cen B & K1V & $5231 \pm 20$ & $4.53 \pm 0.03$ & $0.22 \pm 0.10$ \\
$\epsilon$ Eri & K2Vk: & $5076 \pm 30$ & $4.61 \pm 0.03$ & $-0.09 \pm 0.06$ \\
\hline \multicolumn{5}{c}{ Metal-poor dwarfs (MPD) } \\
HD 49933 & F2V & $6635 \pm 91$ & $4.20 \pm 0.03$ & $-0.41 \pm 0.08$ \\
HD 22879 & F9V & $5868 \pm 89$ & $4.27 \pm 0.04$ & $-0.86 \pm 0.05$ \\
$\tau$ Cet & G8.5V & $5414 \pm 21$ & $4.49 \pm 0.02$ & $-0.49 \pm 0.03$ \\
$\mu$ Cas & G5Vb & $5308 \pm 29$ & $4.41 \pm 0.06$ & $-0.81 \pm 0.03$ \\
\hline \multicolumn{5}{c}{ Metal-rich giants (MRG) } \\
$\beta$ Hyi & G0V & $5873 \pm 45$ & $3.98 \pm 0.02$ & $-0.04 \pm 0.06$ \\
$\xi$ Hya & G7III & $5044 \pm 40$ & $2.87 \pm 0.02$ & $0.16 \pm 0.20$ \\
$\epsilon$ Vir & G8III & $4983 \pm 61$ & $2.77 \pm 0.02$ & $0.15 \pm 0.16$ \\
$\delta$ Eri & K1III-IV & $4954 \pm 30$ & $3.76 \pm 0.02$ & $0.06 \pm 0.05$ \\
$\beta$ Gem & K0IIIb & $4858 \pm 60$ & $2.90 \pm 0.08$ & $0.13 \pm 0.16$ \\
$\mu$ Leo & K2III & $4474 \pm 60$ & $2.51 \pm 0.11$ & $0.25 \pm 0.15$ \\
$\beta$ Ara & K3Ib-II & $4197 \pm 50$ & $1.05 \pm 0.15$ & $-0.05 \pm 0.39$ \\
\hline \multicolumn{5}{c}{ Metal-poor dwarfs (MPG) } \\
$\epsilon$ For & K2V* & $5123 \pm 78$ & $3.52 \pm 0.08$ & $-0.60 \pm 0.10$ \\
HD 107328 & K0IIIb & $4496 \pm 59$ & $2.09 \pm 0.13$ & $-0.33 \pm 0.16$ \\
Arcturus & K1.5III & $4286 \pm 35$ & $1.60 \pm 0.20$ & $-0.52 \pm 0.08$ \\
HD 220009 & K2III & $4217 \pm 60$ & $1.43 \pm 0.12$ & $-0.74 \pm 0.13$ \\
\hline \multicolumn{5}{c}{} \\
\hline \multicolumn{5}{c}{}
\end{tabular}


H. M. Tabernero et al.: StePAR: an automatic code to infer stellar atmospheric parameters

Table A.2. StePAR results.

\begin{tabular}{|c|c|c|c|c|c|c|c|c|c|c|c|}
\hline Star & Spectral type & Source & $S / N$ & $\begin{array}{l}T_{\text {eff }} \\
{[\mathrm{K}]} \\
\end{array}$ & $\begin{array}{c}\Delta T_{\text {eff }} \\
{[\mathrm{K}]} \\
\end{array}$ & $\begin{array}{l}\log g \\
{[\operatorname{dex}]}\end{array}$ & $\begin{array}{c}\Delta \log g \\
{[\operatorname{dex}]}\end{array}$ & $\begin{array}{c}\xi_{\text {micro }} \\
{\left[\mathrm{km} \mathrm{s}^{-1}\right]}\end{array}$ & $\begin{array}{c}\Delta \xi_{\text {micro }} \\
{\left[\mathrm{km} \mathrm{s}^{-1}\right]}\end{array}$ & $\begin{array}{c}{[\mathrm{Fe} / \mathrm{H}]} \\
{[\mathrm{dex}]} \\
\end{array}$ & $\begin{array}{c}\Delta[\mathrm{Fe} / \mathrm{H}] \\
{[\mathrm{dex}]}\end{array}$ \\
\hline \multicolumn{12}{|c|}{ Metal-rich dwarfs (MRD) } \\
\hline Procyon & F5IV-V & NARVAL & 765 & 6710 & 50 & 3.96 & 0.09 & 1.70 & 0.06 & 0.07 & 0.03 \\
\hline Procyon & F5IV-V & UVES.POP & 1016 & 6675 & 52 & 3.90 & 0.09 & 1.63 & 0.06 & 0.04 & 0.03 \\
\hline$\beta$ Vir & F9V & ESPaDOnS & 635 & 6206 & 51 & 4.15 & 0.11 & 1.43 & 0.07 & 0.16 & 0.04 \\
\hline$\beta$ Vir & F9V & NARVAL & 400 & 6188 & 44 & 4.12 & 0.09 & 1.33 & 0.05 & 0.19 & 0.03 \\
\hline$\mu$ Ara & G3IV-V & HARPS.Archive & 252 & 5814 & 61 & 4.27 & 0.14 & 0.95 & 0.09 & 0.38 & 0.05 \\
\hline$\mu$ Ara & G3IV-V & UVES & 309 & 5889 & 64 & 4.41 & 0.13 & 1.03 & 0.10 & 0.38 & 0.05 \\
\hline $18 \mathrm{Sco}$ & $\mathrm{G} 2 \mathrm{Va}$ & ESPaDOnS & 383 & 5807 & 53 & 4.40 & 0.13 & 0.69 & 0.11 & 0.12 & 0.04 \\
\hline $18 \mathrm{Sco}$ & $\mathrm{G} 2 \mathrm{Va}$ & NARVAL & 380 & 5808 & 57 & 4.43 & 0.12 & 0.74 & 0.11 & 0.11 & 0.05 \\
\hline$\alpha$ Cen A & $\mathrm{G} 2 \mathrm{~V}$ & HARPS.Archive & 496 & 5820 & 60 & 4.35 & 0.13 & 0.96 & 0.09 & 0.28 & 0.05 \\
\hline$\alpha$ Cen A & $\mathrm{G} 2 \mathrm{~V}$ & UVES & 316 & 5824 & 66 & 4.35 & 0.14 & 0.86 & 0.11 & 0.30 & 0.05 \\
\hline Sun & $\mathrm{G} 2 \mathrm{~V}$ & HARPS.Archive & 549 & 5748 & 54 & 4.38 & 0.11 & 0.62 & 0.12 & 0.07 & 0.04 \\
\hline Sun & $\mathrm{G} 2 \mathrm{~V}$ & NARVAL & 828 & 5766 & 63 & 4.38 & 0.15 & 0.70 & 0.12 & 0.08 & 0.05 \\
\hline$\alpha$ Cen B & $\mathrm{K} 1 \mathrm{~V}$ & HARPS & 469 & 5088 & 142 & 4.19 & 0.31 & 0.50 & 0.30 & 0.29 & 0.10 \\
\hline$\epsilon$ Eri & K2Vk: & UVES & 220 & 5088 & 101 & 4.41 & 0.29 & 0.78 & 0.21 & -0.05 & 0.06 \\
\hline$\epsilon$ Eri & K2Vk: & UVES.POP & 1653 & 4998 & 100 & 4.40 & 0.23 & 0.41 & 0.28 & 0.00 & 0.06 \\
\hline \multicolumn{12}{|c|}{ Metal-poor dwarfs (MPD) } \\
\hline HD 49933 & $\mathrm{~F} 2 \mathrm{~V}$ & HARPS.Archive & 319 & 6659 & 41 & 4.05 & 0.10 & 1.54 & 0.05 & -0.45 & 0.03 \\
\hline HD 49933 & $\mathrm{~F} 2 \mathrm{~V}$ & ESPaDOnS & 1169 & 6640 & 41 & 4.03 & 0.09 & 1.56 & 0.05 & -0.43 & 0.03 \\
\hline HD 22879 & F9V & HARPS.GBOG & 322 & 5718 & 24 & 3.97 & 0.08 & 1.04 & 0.03 & -0.96 & 0.02 \\
\hline HD 22879 & F9V & NARVAL & 297 & 5720 & 22 & 3.99 & 0.07 & 1.02 & 0.03 & -0.95 & 0.02 \\
\hline$\tau$ Cet & $\mathrm{G} 8.5 \mathrm{~V}$ & ESPaDOnS & 1238 & 5285 & 90 & 4.46 & 0.22 & 0.43 & 0.21 & -0.52 & 0.07 \\
\hline$\tau$ Cet & G8.5V & NARVAL & 357 & 5243 & 138 & 4.37 & 0.34 & 0.50 & 0.35 & -0.54 & 0.12 \\
\hline$\mu$ Cas & $\mathrm{G} 5 \mathrm{Vb}$ & NARVAL & 269 & 5257 & 66 & 4.32 & 0.15 & 0.42 & 0.24 & -0.83 & 0.06 \\
\hline \multicolumn{12}{|c|}{ Metal-rich giants (MRG) } \\
\hline$\beta$ Hyi & G0V & HARPS.Archive & 428 & 5825 & 62 & 3.90 & 0.12 & 0.84 & 0.08 & -0.02 & 0.05 \\
\hline$\beta$ Нyi & G0V & UVES.POP & 676 & 5824 & 65 & 3.89 & 0.12 & 0.92 & 0.07 & -0.05 & 0.05 \\
\hline$\xi$ Hya & G7III & HARPS.GBOG & 391 & 5048 & 71 & 2.87 & 0.23 & 1.17 & 0.07 & 0.20 & 0.06 \\
\hline$\xi$ Hya & G7III & ESPaDOnS & 526 & 5034 & 72 & 2.82 & 0.25 & 1.20 & 0.07 & 0.17 & 0.05 \\
\hline$\epsilon$ Vir & G8III & ESPaDOnS & 435 & 5115 & 69 & 2.83 & 0.23 & 1.33 & 0.07 & 0.24 & 0.06 \\
\hline$\epsilon \mathrm{Vir}$ & G8III & HARPS.GBOG & 392 & 5108 & 74 & 2.85 & 0.23 & 1.33 & 0.07 & 0.24 & 0.06 \\
\hline$\delta$ Eri & K1III-IV & HARPS.Archive & 525 & 5011 & 95 & 3.60 & 0.23 & 0.77 & 0.13 & 0.19 & 0.06 \\
\hline$\delta$ Eri & K1III-IV & UVES.POP & 548 & 4976 & 96 & 3.57 & 0.23 & 0.72 & 0.14 & 0.19 & 0.06 \\
\hline$\beta \mathrm{Gem}$ & K0IIIb & HARPS.GBOG & 370 & 4893 & 98 & 2.99 & 0.27 & 1.07 & 0.09 & 0.25 & 0.06 \\
\hline$\beta \mathrm{Gem}$ & K0IIIb & UVES & 163 & 4864 & 100 & 2.88 & 0.32 & 1.14 & 0.10 & 0.18 & 0.07 \\
\hline$\mu$ Leo & K2III & ESPaDOnS & 779 & 4518 & 168 & 2.19 & 0.57 & 1.25 & 0.12 & 0.37 & 0.13 \\
\hline$\mu$ Leo & K2III & NARVAL & 402 & 4516 & 166 & 2.24 & 0.55 & 1.34 & 0.13 & 0.32 & 0.12 \\
\hline$\beta$ Ara & K3Ib-II & HARPS.GBOG & 414 & 4413 & 164 & 1.38 & 0.65 & 2.20 & 0.18 & 0.03 & 0.14 \\
\hline \multicolumn{12}{|c|}{ Metal-poor giants (MPD) } \\
\hline$\epsilon$ For & $\mathrm{K} 2 \mathrm{~V}^{*}$ & HARPS.GBOG & 334 & 5092 & 54 & 3.63 & 0.15 & 0.70 & 0.10 & -0.53 & 0.04 \\
\hline HD 107328 & K0IIIb & HARPS.GBOG & 459 & 4418 & 62 & 1.85 & 0.26 & 1.71 & 0.07 & -0.43 & 0.06 \\
\hline HD 107328 & KOIIIb & NARVAL & 375 & 4442 & 60 & 1.98 & 0.25 & 1.68 & 0.06 & -0.39 & 0.06 \\
\hline Arcturus & K1.5III & HARPS.Archive & 475 & 4337 & 60 & 1.87 & 0.25 & 1.64 & 0.07 & -0.44 & 0.06 \\
\hline Arcturus & K1.5III & UVES.POP & 1208 & 4338 & 56 & 1.83 & 0.24 & 1.59 & 0.06 & -0.44 & 0.05 \\
\hline HD 220009 & K2III & HARPS.GBOG & 347 & 4346 & 46 & 1.79 & 0.20 & 1.42 & 0.05 & -0.65 & 0.04 \\
\hline HD 220009 & K2III & NARVAL & 376 & 4346 & 54 & 1.93 & 0.24 & 1.45 & 0.06 & -0.63 & 0.05 \\
\hline
\end{tabular}


Table A.3. Merged Fe I linelists.

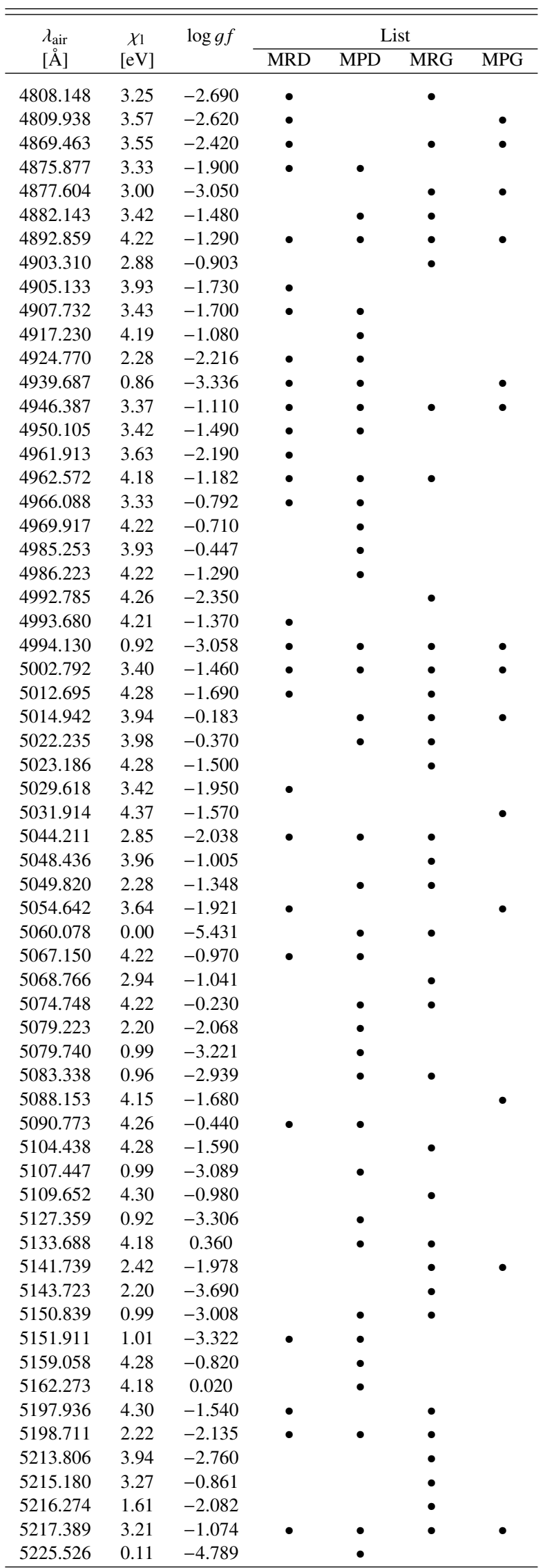

Table A.3. continued.

\begin{tabular}{|c|c|c|c|c|c|c|}
\hline \multirow{2}{*}{$\begin{array}{l}\lambda_{\text {air }} \\
[\AA]]\end{array}$} & \multirow{2}{*}{$\begin{array}{c}\chi_{1} \\
{[\mathrm{eV}]}\end{array}$} & \multirow[t]{2}{*}{$\log g f$} & \multicolumn{4}{|c|}{ List } \\
\hline & & & MRD & MPD & MRG & MPG \\
\hline 5228.376 & 4.22 & -1.190 & & - & & \\
\hline 5229.845 & 3.28 & -0.967 & & $\bullet$ & & \\
\hline 5242.491 & 3.63 & -0.967 & - & - & - & - \\
\hline 5243.776 & 4.26 & -1.050 & - & - & - & - \\
\hline 5247.050 & 0.09 & -4.949 & $\bullet$ & $\bullet$ & & \\
\hline 5250.209 & 0.12 & -4.933 & & - & & \\
\hline 5250.646 & 2.20 & -2.180 & & - & & \\
\hline 5253.462 & 3.28 & -1.579 & & - & - & \\
\hline 5285.127 & 4.44 & -1.660 & $\bullet$ & & $\bullet$ & $\bullet$ \\
\hline 5288.525 & 3.70 & -1.493 & $\bullet$ & & & $\bullet$ \\
\hline 5293.959 & 4.14 & -1.770 & & & - & - \\
\hline 5294.547 & 3.64 & -2.760 & - & & - & \\
\hline 5295.312 & 4.42 & -1.590 & $\bullet$ & & $\bullet$ & $\bullet$ \\
\hline 5307.361 & 1.61 & -2.912 & $\bullet$ & $\bullet$ & & $\bullet$ \\
\hline 5321.108 & 4.44 & -1.089 & & & & $\bullet$ \\
\hline 5322.041 & 2.28 & -2.802 & $\bullet$ & & & $\bullet$ \\
\hline 5339.929 & 3.27 & -0.635 & & & & - \\
\hline 5364.871 & 4.45 & 0.228 & & & - & - \\
\hline 5373.709 & 4.47 & -0.710 & $\bullet$ & $\bullet$ & & \\
\hline 5379.574 & 3.70 & -1.514 & $\bullet$ & - & $\bullet$ & $\bullet$ \\
\hline 5386.333 & 4.15 & -1.670 & - & & - & - \\
\hline 5389.479 & 4.42 & -0.410 & & - & - & \\
\hline 5397.618 & 3.63 & -2.528 & & & - & \\
\hline 5398.279 & 4.45 & -0.630 & $\bullet$ & $\bullet$ & $\bullet$ & \\
\hline 5400.501 & 4.37 & -0.160 & - & - & & \\
\hline 5401.266 & 4.32 & -1.820 & - & & - & \\
\hline 5409.133 & 4.37 & -1.200 & - & $\bullet$ & & \\
\hline 5417.033 & 4.42 & -1.580 & - & & $\bullet$ & \\
\hline 5424.068 & 4.32 & 0.520 & & - & & \\
\hline 5436.295 & 4.39 & -1.440 & $\bullet$ & & $\bullet$ & $\bullet$ \\
\hline 5436.588 & 2.28 & -2.964 & & & $\bullet$ & \\
\hline 5441.339 & 4.31 & -1.630 & - & & - & - \\
\hline 5445.042 & 4.39 & -0.020 & & - & - & - \\
\hline 5460.873 & 3.07 & -3.426 & & & $\bullet$ & $\bullet$ \\
\hline 5461.550 & 4.45 & -1.800 & $\bullet$ & & - & \\
\hline 5463.275 & 4.44 & 0.070 & & & - & - \\
\hline 5464.280 & 4.14 & -1.402 & & & - & \\
\hline 5466.396 & 4.37 & -0.630 & - & - & & - \\
\hline 5470.093 & 4.45 & -1.710 & $\bullet$ & & $\bullet$ & \\
\hline 5472.709 & 4.21 & -1.495 & - & & & \\
\hline 5473.900 & 4.15 & -0.720 & $\bullet$ & $\bullet$ & & \\
\hline 5483.099 & 4.15 & -1.392 & - & & & $\bullet$ \\
\hline 5501.465 & 0.96 & -3.046 & & - & & \\
\hline 5506.779 & 0.99 & -2.795 & & $\bullet$ & & \\
\hline 5522.446 & 4.21 & -1.450 & $\bullet$ & $\bullet$ & & \\
\hline 5536.580 & 2.83 & -3.710 & & & & - \\
\hline 5539.280 & 3.64 & -2.560 & & & & - \\
\hline 5543.147 & 3.70 & -1.470 & & $\bullet$ & & \\
\hline 5543.936 & 4.22 & -1.040 & $\bullet$ & - & - & - \\
\hline 5546.506 & 4.37 & -1.210 & & - & & \\
\hline 5549.949 & 3.70 & -2.810 & & & - & \\
\hline 5554.894 & 4.55 & -0.270 & & - & & \\
\hline 5560.212 & 4.44 & -1.090 & $\bullet$ & $\bullet$ & $\bullet$ & \\
\hline 5572.842 & 3.40 & -0.289 & & & & $\bullet$ \\
\hline 5576.089 & 3.43 & -0.900 & & - & - & - \\
\hline 5618.632 & 4.21 & -1.255 & $\bullet$ & $\bullet$ & $\bullet$ & $\bullet$ \\
\hline 5619.595 & 4.39 & -1.600 & $\bullet$ & & & $\bullet$ \\
\hline 5633.946 & 4.99 & -0.230 & & - & & \\
\hline 5635.822 & 4.26 & -1.790 & $\bullet$ & & & \\
\hline 5636.696 & 3.64 & -2.510 & - & & - & \\
\hline 5638.262 & 4.22 & -0.720 & - & - & & \\
\hline 5641.434 & 4.26 & -1.080 & - & . & & \\
\hline
\end{tabular}


H. M. Tabernero et al.: STEPAR: an automatic code to infer stellar atmospheric parameters

Table A.3. continued.

\begin{tabular}{|c|c|c|c|c|c|c|}
\hline \multirow{2}{*}{$\begin{array}{l}\lambda_{\text {air }} \\
[\AA]]\end{array}$} & \multirow{2}{*}{$\begin{array}{c}\chi_{1} \\
{[\mathrm{eV}]}\end{array}$} & \multirow[t]{2}{*}{$\log g f$} & \multicolumn{4}{|c|}{ List } \\
\hline & & & MRD & MPD & MRG & MPG \\
\hline 5649.987 & 5.10 & -0.820 & • & & & • \\
\hline 5651.469 & 4.47 & -1.900 & $\bullet$ & & & \\
\hline 5652.318 & 4.26 & -1.850 & $\bullet$ & & & \\
\hline 5653.865 & 4.39 & -1.540 & $\bullet$ & & & • \\
\hline 5655.176 & 5.06 & -0.600 & • & & & • \\
\hline 5661.345 & 4.28 & -1.756 & $\bullet$ & & & $\bullet$ \\
\hline 5662.516 & 4.18 & -0.447 & • & • & & $\bullet$ \\
\hline 5679.023 & 4.65 & -0.820 & $\bullet$ & $\bullet$ & $\bullet$ & $\bullet$ \\
\hline 5691.497 & 4.30 & -1.450 & & $\bullet$ & & \\
\hline 5696.089 & 4.55 & -1.720 & $\bullet$ & & & \\
\hline 5701.544 & 2.56 & -2.193 & $\bullet$ & $\bullet$ & & \\
\hline 5705.464 & 4.30 & -1.355 & $\bullet$ & & & \\
\hline 5717.833 & 4.28 & -0.990 & $\bullet$ & $\bullet$ & $\bullet$ & • \\
\hline 5720.886 & 4.55 & -1.631 & $\bullet$ & & $\bullet$ & $\bullet$ \\
\hline 5731.762 & 4.26 & -1.200 & • & $\bullet$ & & $\bullet$ \\
\hline 5732.296 & 4.99 & -1.460 & • & & & \\
\hline 5741.848 & 4.26 & -1.672 & $\bullet$ & & & \\
\hline 5759.262 & 4.65 & -2.216 & & & $\bullet$ & \\
\hline 5778.453 & 2.59 & -3.430 & & & $\bullet$ & \\
\hline 5784.658 & 3.40 & -2.547 & & & $\bullet$ & \\
\hline 5844.918 & 4.15 & -3.054 & & & • & \\
\hline 5849.683 & 3.70 & -2.890 & & & & $\bullet$ \\
\hline 5852.219 & 4.55 & -1.230 & • & & $\bullet$ & \\
\hline 5853.148 & 1.49 & -5.180 & & & - & • \\
\hline 5855.076 & 4.61 & -1.478 & $\bullet$ & & $\bullet$ & \\
\hline 5856.088 & 4.29 & -1.327 & & & • & \\
\hline 5858.778 & 4.22 & -2.160 & & & - & \\
\hline 5861.109 & 4.28 & -2.304 & & & $\bullet$ & • \\
\hline 5883.816 & 3.96 & -1.260 & • & - & & • \\
\hline 5902.473 & 4.59 & -1.710 & & & & $\bullet$ \\
\hline 5905.671 & 4.65 & -0.690 & $\bullet$ & $\bullet$ & • & - \\
\hline 5909.972 & 3.21 & -2.587 & $\bullet$ & & $\bullet$ & • \\
\hline 5916.247 & 2.45 & -2.994 & $\bullet$ & & $\bullet$ & $\bullet$ \\
\hline 5927.789 & 4.65 & -0.990 & $\bullet$ & & $\bullet$ & \\
\hline 5929.676 & 4.55 & -1.310 & • & & $\bullet$ & \\
\hline 5930.180 & 4.65 & -0.230 & $\bullet$ & $\bullet$ & $\bullet$ & \\
\hline 5934.654 & 3.93 & -1.070 & $\bullet$ & $\bullet$ & & $\bullet$ \\
\hline 5940.991 & 4.18 & -2.050 & & & & • \\
\hline 5952.718 & 3.98 & -1.340 & & & & $\bullet$ \\
\hline 5956.694 & 0.86 & -4.599 & $\bullet$ & • & - & • \\
\hline 6003.011 & 3.88 & -1.100 & $\bullet$ & $\bullet$ & $\bullet$ & $\bullet$ \\
\hline 6012.210 & 2.22 & -4.038 & & & & • \\
\hline 6019.365 & 3.57 & -3.310 & & & & $\bullet$ \\
\hline 6024.057 & 4.55 & -0.120 & • & • & & $\bullet$ \\
\hline 6027.051 & 4.08 & -1.089 & $\bullet$ & $\bullet$ & $\bullet$ & $\bullet$ \\
\hline 6056.005 & 4.73 & -0.320 & & $\bullet$ & & \\
\hline 6065.482 & 2.61 & -1.529 & & $\bullet$ & $\bullet$ & \\
\hline 6079.008 & 4.65 & -1.020 & $\bullet$ & $\bullet$ & $\bullet$ & $\bullet$ \\
\hline 6082.710 & 2.22 & -3.576 & • & & $\bullet$ & $\bullet$ \\
\hline 6093.643 & 4.61 & -1.400 & $\bullet$ & & $\bullet$ & $\bullet$ \\
\hline 6094.373 & 4.65 & -1.840 & & & & $\bullet$ \\
\hline 6096.664 & 3.98 & -1.830 & $\bullet$ & & & $\bullet$ \\
\hline 6098.244 & 4.56 & -1.859 & $\bullet$ & & - & $\bullet$ \\
\hline 6120.246 & 0.92 & -5.970 & & & $\bullet$ & $\bullet$ \\
\hline 6127.906 & 4.14 & -1.399 & $\bullet$ & $\bullet$ & & • \\
\hline 6136.615 & 2.45 & -1.402 & & $\bullet$ & & \\
\hline 6136.994 & 2.20 & -2.950 & & • & & \\
\hline 6137.691 & 2.59 & -1.402 & & $\bullet$ & & \\
\hline 6151.617 & 2.18 & -3.295 & $\bullet$ & $\bullet$ & & $\bullet$ \\
\hline 6165.360 & 4.14 & -1.473 & $\bullet$ & $\bullet$ & & $\bullet$ \\
\hline 6170.506 & 4.80 & -0.440 & $\bullet$ & $\bullet$ & & \\
\hline 6173.334 & 2.22 & -2.880 & $\bullet$ & $\bullet$ & $\bullet$ & • \\
\hline 6180.203 & 2.73 & -2.591 & • & & & \\
\hline
\end{tabular}

Table A.3. continued.

\begin{tabular}{|c|c|c|c|c|c|c|}
\hline \multirow{2}{*}{$\begin{array}{l}\lambda_{\text {air }} \\
{[\AA]}\end{array}$} & \multirow{2}{*}{$\begin{array}{c}\chi_{1} \\
{[\mathrm{eV}]}\end{array}$} & \multirow[t]{2}{*}{$\log g f$} & \multicolumn{4}{|c|}{ List } \\
\hline & & & MRD & MPD & MRG & MPG \\
\hline 6187.989 & 3.94 & -1.620 & $\bullet$ & & $\bullet$ & $\bullet$ \\
\hline 6191.557 & 2.43 & -1.416 & & $\bullet$ & & \\
\hline 6199.506 & 2.56 & -4.430 & & & & $\bullet$ \\
\hline 6200.312 & 2.61 & -2.433 & $\bullet$ & $\bullet$ & & $\bullet$ \\
\hline 6213.429 & 2.22 & -2.481 & $\bullet$ & $\bullet$ & & $\bullet$ \\
\hline 6219.280 & 2.20 & -2.432 & $\bullet$ & $\bullet$ & & $\bullet$ \\
\hline 6220.780 & 3.88 & -2.058 & & & & $\bullet$ \\
\hline 6226.734 & 3.88 & -2.120 & $\bullet$ & & & \\
\hline 6229.226 & 2.85 & -2.805 & $\bullet$ & & $\bullet$ & $\bullet$ \\
\hline 6230.722 & 2.56 & -1.281 & $\bullet$ & $\bullet$ & & \\
\hline 6240.646 & 2.22 & -3.230 & $\bullet$ & $\bullet$ & $\bullet$ & $\bullet$ \\
\hline 6246.318 & 3.60 & -0.771 & $\bullet$ & $\bullet$ & $\bullet$ & \\
\hline 6252.555 & 2.40 & -1.699 & $\bullet$ & $\bullet$ & $\bullet$ & $\bullet$ \\
\hline 6265.132 & 2.18 & -2.550 & $\bullet$ & $\bullet$ & $\bullet$ & $\bullet$ \\
\hline 6270.223 & 2.86 & -2.470 & $\bullet$ & & $\bullet$ & $\bullet$ \\
\hline 6271.278 & 3.33 & -2.703 & & & $\bullet$ & $\bullet$ \\
\hline 6280.617 & 0.86 & -4.390 & & $\bullet$ & & \\
\hline 6290.543 & 2.59 & -4.330 & & & & $\bullet$ \\
\hline 6297.793 & 2.22 & -2.737 & & $\bullet$ & & $\bullet$ \\
\hline 6301.500 & 3.65 & -0.720 & & $\bullet$ & $\bullet$ & $\bullet$ \\
\hline 6311.499 & 2.83 & -3.141 & $\bullet$ & & & $\bullet$ \\
\hline 6315.811 & 4.08 & -1.630 & $\bullet$ & & & $\bullet$ \\
\hline 6322.685 & 2.59 & -2.430 & $\bullet$ & $\bullet$ & $\bullet$ & $\bullet$ \\
\hline 6335.330 & 2.20 & -2.177 & $\bullet$ & $\bullet$ & $\bullet$ & $\bullet$ \\
\hline 6336.823 & 3.69 & -0.852 & $\bullet$ & $\bullet$ & $\bullet$ & $\bullet$ \\
\hline 6338.876 & 4.80 & -0.960 & $\bullet$ & & & \\
\hline 6344.148 & 2.43 & -2.919 & & $\bullet$ & & \\
\hline 6355.028 & 2.85 & -2.340 & & $\bullet$ & & \\
\hline 6380.743 & 4.19 & -1.375 & $\bullet$ & & & $\bullet$ \\
\hline 6393.600 & 2.43 & -1.452 & & $\bullet$ & $\bullet$ & \\
\hline 6400.317 & 0.92 & -4.318 & $\bullet$ & $\bullet$ & & \\
\hline 6411.648 & 3.65 & -0.596 & & $\bullet$ & & \\
\hline 6421.350 & 2.28 & -2.012 & $\bullet$ & $\bullet$ & & \\
\hline 6430.845 & 2.18 & -2.005 & $\bullet$ & $\bullet$ & & \\
\hline 6469.192 & 4.84 & -0.730 & $\bullet$ & $\bullet$ & & \\
\hline 6475.624 & 2.56 & -2.941 & $\bullet$ & $\bullet$ & & \\
\hline 6481.870 & 2.28 & -2.981 & $\bullet$ & $\bullet$ & & $\bullet$ \\
\hline 6494.980 & 2.40 & -1.268 & & $\bullet$ & & \\
\hline 6495.741 & 4.84 & -0.840 & $\bullet$ & & & \\
\hline 6496.466 & 4.80 & -0.530 & $\bullet$ & $\bullet$ & & $\bullet$ \\
\hline 6498.938 & 0.96 & -4.687 & $\bullet$ & & & $\bullet$ \\
\hline 6518.366 & 2.83 & -2.438 & & & & $\bullet$ \\
\hline 6533.928 & 4.56 & -1.360 & $\bullet$ & $\bullet$ & $\bullet$ & $\bullet$ \\
\hline 6546.238 & 2.76 & -1.536 & & $\bullet$ & $\bullet$ & $\bullet$ \\
\hline 6574.227 & 0.99 & -5.004 & $\bullet$ & & & \\
\hline 6581.209 & 1.49 & -4.679 & & & & $\bullet$ \\
\hline 6591.313 & 4.59 & -2.081 & & & $\bullet$ & $\bullet$ \\
\hline 6592.912 & 2.73 & -1.473 & $\bullet$ & $\bullet$ & & $\bullet$ \\
\hline 6593.869 & 2.43 & -2.420 & $\bullet$ & $\bullet$ & & $\bullet$ \\
\hline 6597.559 & 4.80 & -0.970 & $\bullet$ & & & $\bullet$ \\
\hline 6608.025 & 2.28 & -3.930 & $\bullet$ & & & $\bullet$ \\
\hline 6609.110 & 2.56 & -2.691 & $\bullet$ & $\bullet$ & & $\bullet$ \\
\hline 6627.544 & 4.55 & -1.590 & $\bullet$ & & & \\
\hline 6633.412 & 4.84 & -1.390 & $\bullet$ & & & \\
\hline 6633.749 & 4.56 & -0.799 & $\bullet$ & & & \\
\hline 6648.080 & 1.01 & -5.918 & & & & $\bullet$ \\
\hline 6703.566 & 2.76 & -3.060 & $\bullet$ & & $\bullet$ & \\
\hline 6710.318 & 1.49 & -4.764 & $\bullet$ & & & \\
\hline 6713.743 & 4.80 & -1.500 & $\bullet$ & & $\bullet$ & \\
\hline 6716.236 & 4.58 & -1.836 & $\bullet$ & & & \\
\hline 6725.356 & 4.10 & -2.100 & & & $\bullet$ & $\bullet$ \\
\hline 6750.151 & 2.42 & -2.618 & $\bullet$ & $\bullet$ & $\bullet$ & $\bullet$ \\
\hline 6752.707 & 4.64 & -1.204 & $\bullet$ & & & \\
\hline
\end{tabular}


Table A.4. Merged Fe II linelists.

\begin{tabular}{ccccccc}
\hline \hline \multirow{2}{*}{$\lambda_{\text {air }}$} & \multirow{2}{*}{$\chi_{1}$} & \multirow{2}{*}{$\log g f$} & \multicolumn{5}{c}{ List } \\
\cline { 5 - 7 }$[\AA]$ & {$[\mathrm{eV}]$} & & MRD & MPD & MRG & MPG \\
\hline 4993.350 & 2.81 & -3.684 & $\bullet$ & $\bullet$ & $\bullet$ & \\
5197.568 & 3.23 & -2.220 & $\bullet$ & $\bullet$ & $\bullet$ & \\
5234.623 & 3.22 & -2.180 & $\bullet$ & $\bullet$ & $\bullet$ & $\bullet$ \\
5256.932 & 2.89 & -4.182 & $\bullet$ & & $\bullet$ & \\
5264.802 & 3.23 & -3.130 & & $\bullet$ & & \\
5284.103 & 2.89 & -3.195 & & $\bullet$ & & \\
5325.552 & 3.22 & -3.160 & & $\bullet$ & & $\bullet$ \\
5414.070 & 3.22 & -3.580 & & & $\bullet$ & \\
5425.248 & 3.20 & -3.220 & $\bullet$ & $\bullet$ & $\bullet$ & $\bullet$ \\
5534.838 & 3.25 & -2.865 & & $\bullet$ & & \\
5991.371 & 3.15 & -3.647 & $\bullet$ & & & \\
6084.102 & 3.20 & -3.881 & $\bullet$ & & $\bullet$ & \\
6149.246 & 3.89 & -2.841 & $\bullet$ & & $\bullet$ & $\bullet$ \\
6238.386 & 3.89 & -2.600 & & $\bullet$ & $\bullet$ & \\
6247.557 & 3.89 & -2.435 & & $\bullet$ & & \\
6369.459 & 2.89 & -4.110 & $\bullet$ & & & $\bullet$ \\
6416.919 & 3.89 & -2.877 & $\bullet$ & & & \\
6432.676 & 2.89 & -3.570 & $\bullet$ & $\bullet$ & $\bullet$ & \\
6456.380 & 3.90 & -2.185 & & $\bullet$ & $\bullet$ & \\
6516.077 & 2.89 & -3.310 & $\bullet$ & $\bullet$ & & \\
\hline
\end{tabular}

